TRANSACTIONS OF THE

AMERICAN MATHEMATICAL SOCIETY

Volume 358, Number 6 , Pages 2695-2711

S 0002-9947(06)03841-4

Article electronically published on January 24, 2006

\title{
SYMMETRY AND INVERSE-CLOSEDNESS OF MATRIX ALGEBRAS AND FUNCTIONAL CALCULUS FOR INFINITE MATRICES
}

\author{
KARLHEINZ GRÖCHENIG AND MICHAEL LEINERT
}

\begin{abstract}
We investigate the symbolic calculus for a large class of matrix algebras that are defined by the off-diagonal decay of infinite matrices. Applications are given to the symmetry of some highly non-commutative Banach algebras, to the analysis of twisted convolution, and to the theory of localized frames.
\end{abstract}

\section{INTRODUCTION}

The purpose of this paper is twofold: on the one hand, we develop a functional calculus for various classes of infinite matrices with off-diagonal decay, and on the other hand, we construct explicit examples of symmetric highly non-commutative involutive Banach algebras.

Our point of departure is two important results about the properties of inverse matrices. Assume that $A$ is an infinite matrix that is bounded on $\ell^{2}(\mathbb{Z})$ with bounded inverse. If either $a_{k l}=0$ for $|k-l| \geq M$ ( $A$ is a banded matrix) or if $\left|a_{k l}\right|=$ $\mathcal{O}\left(e^{-\alpha|k-l|}\right)$ (exponential off-diagonal decay), then the inverse matrix $B=A^{-1}$ satisfies $\left|b_{k l}\right|=\mathcal{O}\left(e^{-\beta|k-l|}\right)$ for some $\beta, 0<\beta<\alpha$. See [12, 24, 30] for a few versions of this statement.

Similarly Jaffard's Theorem [24] says that if $A$ is boundedly invertible on $\ell^{2}\left(\mathbb{Z}^{d}\right)$ and $\left|a_{k l}\right|=\mathcal{O}\left(|k-l|^{-s}\right)$ for some $s>d$, then its inverse $B=A^{-1}$ has the same polynomial-type off-diagonal decay $\left|b_{k l}\right|=\mathcal{O}\left(|k-l|^{-s}\right)$. This result is even more striking, because the order of polynomial decay is preserved exactly, whereas the order of exponential decay is not preserved in general.

Both types of results are highly relevant and have numerous applications in numerical analysis [9, 12, 33, 35], wavelet theory [24, time-frequency analysis [5, 17], and sampling theory [1, 19, to mention just a few non-trivial applications.

In these problems it seems desirable to treat decay conditions that are intermediate between polynomial decay (too slow) and exponential decay (too fast, and not exactly preserved). In this paper we treat so-called "subexponential" decay conditions and prove several versions of Jaffard's Theorem for a large class of decay conditions. We give examples to show that our results are sharp.

Received by the editors November 4, 2003 and, in revised form, August 13, 2004 2000 Mathematics Subject Classification. Primary 47B37, 47A60, 46H30, 42C15.

Key words and phrases. Off-diagonal decay of matrices, weight function, invertibility of operators, invariance of spectrum, symmetric Banach algebras, Gelfand-Raikov-Shilov condition, Schur's test, twisted convolution, localized frames. 
From a different point of view, Jaffard's Lemma can be viewed as a functional calculus for certain matrix algebras. The statements resemble the Wiener-Levy theorem for absolutely convergent Fourier series, but now they are for extremely non-commutative algebras. In this context it seems natural to approach the problem with Banach algebra techniques and the notion of symmetric involutive algebras. Yet Jaffard's ingenious proof is purely analytical, using commutator estimates and a bootstrap argument. Despite its beauty and depth, the original proof cannot be adapted to other decay conditions, because it uses special properties of polynomial weights. While estimates of spectral radii occur implicitly in 24], no reference to Banach algebras is made. In this paper we develop the necessary Banach algebra techniques to extend Jaffard's Theorem to subexponential decay of matrices. As a by-product, we obtain a new and more algebraic proof of Jaffard's Lemma.

Our second motivation concerns the concept of symmetry and inverse closedness of Banach algebras. Here an involutive algebra is symmetric if the spectrum of positive elements is positive. While there are many abstract results about symmetric Banach algebras (see [4, 27, 28, and references cited there), it is often extremely difficult to verify the symmetry of specific examples. The recent breakthrough by Losert 25 has renewed interest in understanding the symmetry of specific classes of involutive Banach algebras. Solving a 30-year old conjecture, Losert 25] succeeded in showing that the group algebra of a compactly generated, locally compact group of polynomial growth is symmetric. Subsequently, we have developed techniques to verify the symmetry of weighted $L^{1}$-algebras on locally compact groups of polynomial growth [15] and of Banach algebras of twisted convolution [20].

Our main results about the functional calculus with infinite matrices can also be interpreted as statements about the symmetry of a large class of matrix algebras. In a sense they extend our results for locally compact groups [15] to Banach algebras of operators which have much less structure. In this regard, Barnes's results on Banach algebras of integral operators $[3$ need to be mentioned. These are in a similar spirit, but unfortunately they work only for sublinear weights.

We expect our main results to be useful in numerical applications. Theorems 6 and 10 help unify, refine, and improve all those results where previously Jaffard's Lemma had been used. In Section 5 we discuss two mathematical problems where our main theorems either lead to significant simplification or to conceptual progress.

First, we briefly study the spectrum of twisted convolution operators and prove a (non-commutative) version of Wiener's Lemma. Based on our main theorem, we give a new, concise, and much shorter proof of Wiener's Lemma for twisted convolution. This statement has been instrumental for the construction of Gabor frames with good time-frequency concentration and the solution of a conjecture of Janssen and Feichtinger [20].

Second, we offer an new treatment of localized frames and their duals. A new definition of localization highlights the role of Banach algebras in frame theory and allows for a conceptually much simpler approach to localized frames.

Furthermore, one could revisit other topics where off-diagonal decay of inverse matrices is crucial. We mention Jaffard's "lemme de fenetre" and perturbation theory [24, the finite section method for operator equations [21, 35] and for frames [9], local error estimates of wavelet and Gabor expansions [1], or the time-frequency decay of dual Gabor windows [17. These applications were orginally formulated 
with polynomial decay conditions; in all of them, one may substitute Jaffard's Theorem by Theorems 6 or 10 and then obtain refinements of existing results (with subexponential decay functions) that had not been available before.

Finally, let us mention that the main results could also be stated for integral operators on suitable measure spaces. Since only technicalities and not new ideas are required, we will not state these results explicitly.

The paper is organized as follows: In Section 2 we collect background on weight functions used to quantify off-diagonal decay of matrices and present the main tools from Banach algebra theory. In Section 3 we give the first version of a functional calculus for Banach algebras of matrices that are defined by Schur-type conditions. These are technically easier to treat than decay conditions. The treatment of decay conditions is then carried out in Section 4 and is based on the main result of Section 3. In the final Section 5 we discuss two applications to twisted convolution and to localized frames.

\section{DeCAY CONDitions AND MATRIX ALGEBRAS}

2.1. Weights. A weight $v$ is a non-negative function on $\mathbb{R}^{d}$. For the study of decay conditions of matrices it is natural to impose the following additional conditions:

(a) Let $\|\cdot\|$ be a norm on $\mathbb{R}^{d}$ and let $\rho:[0, \infty) \longrightarrow[0, \infty)$ be a continuous concave function, normalized by $\rho(0)=0$. Then $v$ is of the form

$$
v(x)=e^{\rho(\|x\|)} .
$$

Then $v$ satisfies $v(0)=1, v(x)=v(-x)$ and $v$ is submultiplicative, i.e.,

$$
v(x+y) \leq v(x) v(y) .
$$

(b) $v$ satisfies the GRS-condition (Gelfand-Raikov-Shilov condition [16])

$$
\lim _{n \rightarrow \infty} v(n x)^{1 / n}=1 \quad \text { for all } x \in \mathbb{R}^{d} .
$$

Equivalently, we have

$$
\lim _{\xi \rightarrow \infty} \frac{\rho(\xi)}{\xi}=0 .
$$

We will call a weight satisfying these conditions an admissible weight and henceforth use only such weights. Note that unless $v$ (and thus $\rho$ ) is bounded, $\rho$ must be strictly increasing as a consequence of its concavity property.

In the following we will always assume that the weight $v$ is defined on $\mathbb{R}^{d}$, although we mainly use the restriction of $v$ to $\mathbb{Z}^{d}$.

Examples. The typical examples of admissible weights are the polynomial weights $v_{s}(x)=(1+|x|)^{s}$ for $s \geq 0$ and the subexponential weights of the form $v(x)=e^{\alpha|x|^{\beta}}$ for $\alpha>0$ and $0<\beta<1$. More general weights are mixtures of the form

$$
v(x)=e^{\alpha|x|^{\beta}}(1+|x|)^{s}(\log (e+|x|))^{t},
$$

where $\alpha \geq 0,0 \leq \beta<1, s \geq 0$, and $t \geq 0$.

For later use we remark that the polynomial weights $\tau_{s}(x)=(1+|x|)^{s}, s \geq 0$, are also "weakly subadditive". This means that there is a $\kappa=\kappa(s)$ (and without loss of generality we may assume that $\kappa \geq 1$ ) such that

$$
\tau_{s}(x+y) \leq \kappa\left(\tau_{s}(x)+\tau_{s}(y)\right) \quad x, y \in \mathbb{R}^{d} .
$$


2.2. Matrix algebras. We now introduce classes of infinite matrices defined by the off-diagonal decay of their entries. The decay will be quantified by an admissible weight function $v$ on $\mathbb{Z}^{d}$.

Definition 1. The class $\mathcal{A}_{v}^{1}$ consists of all matrices $A=\left(a_{k l}\right)_{k, l \in \mathbb{Z}^{d}}$ such that

$$
\sup _{k \in \mathbb{Z}^{d}} \sum_{l \in \mathbb{Z}^{d}}\left|a_{k l}\right| v(k-l)<\infty \quad \text { and } \quad \sup _{l \in \mathbb{Z}^{d}} \sum_{k \in \mathbb{Z}^{d}}\left|a_{k l}\right| v(k-l)<\infty
$$

with norm

$$
\|A\|_{\mathcal{A}_{v}^{1}}=\max \left\{\sup _{k} \sum_{l \in \mathbb{Z}^{d}}\left|a_{k l}\right| v(k-l), \sup _{l} \sum_{k \in \mathbb{Z}^{d}}\left|a_{k l}\right| v(k-l)\right\} .
$$

We write $\mathcal{A}^{1}$ in the case of the trivial weight $v \equiv 1$. In this notation the standard Schur test states that if $A \in \mathcal{A}^{1}$, then $A$ is bounded on all $\ell^{p}\left(\mathbb{Z}^{d}\right)$ for $1 \leq p \leq \infty$; see, e.g., [17, L. 6.2.1].

Definition 2. The class $\mathcal{A}_{v}$ consists of all matrices $A=\left(a_{k l}\right)_{k, l \in \mathbb{Z}^{d}}$ such that, for some $C>0$,

$$
\left|a_{k l}\right| \leq C v(k-l),^{-1} \quad \forall k, l \in \mathbb{Z}^{d} .
$$

A Banach space norm on $\mathcal{A}_{v}$ is given by

$$
\|A\|_{\mathcal{A}_{v}}=\sup _{k, l \in \mathbb{Z}^{d}}\left|a_{k l}\right| v(k-l) .
$$

Lemma 1. (a) $\mathcal{A}_{v}^{1}$ (and $\mathcal{A}^{1}$ ) is always an involutive Banach algebra of bounded operators acting on $\ell^{2}\left(\mathbb{Z}^{d}\right)$. The involution is given by the adjoint matrix $A^{*}$ with entries $\left(A^{*}\right)_{k l}=\overline{a_{l k}}$ and is an isometry on $\mathcal{A}_{v}^{1}$ and $\mathcal{A}^{1}$.

(b) If $\sum_{k \in \mathbb{Z}^{d}} v(k)^{-1}<\infty$, then $\mathcal{A}_{v} \subseteq \mathcal{A}^{1}$. If in addition $v$ satisfies $v^{-1} * v^{-1} \leq$ $C v^{-1}$ (such $a v$ is called subconvolutive [13]), then $\mathcal{A}_{v}$ is an algebra of bounded operators on $\ell^{2}\left(\mathbb{Z}^{d}\right)$.

Proof. By Schur's test $\mathcal{A}^{1}$ consists exactly of those matrices that are bounded simultaneously on $\ell^{1}\left(\mathbb{Z}^{d}\right)$ and $\ell^{\infty}\left(\mathbb{Z}^{d}\right)$ (and hence on $\ell^{2}\left(\mathbb{Z}^{d}\right)$ ). The $\mathcal{A}^{1}$-norm is the larger of the operator norms on $\ell^{1}$ or on $\ell^{\infty}$ and thus $\mathcal{A}^{1}$ is a Banach algebra.

For the weighted case we use the submultiplicativity in the form $v(k-l) \leq$ $v(k-j) v(j-l)$ and estimate for $A, B \in \mathcal{A}_{v}^{1}$ that

$$
\begin{aligned}
\sum_{l \in \mathbb{Z}^{d}}\left|(A B)_{k l}\right| v(k-l) & =\sum_{l \in \mathbb{Z}^{d}}\left|\sum_{j \in \mathbb{Z}^{d}} a_{k j} b_{j l}\right| v(k-l) \\
& \leq \sum_{l \in \mathbb{Z}^{d}} \sum_{j \in \mathbb{Z}^{d}}\left|a_{k j}\right|\left|b_{j l}\right| v(k-j) v(j-l) \\
& \leq \sum_{j \in \mathbb{Z}^{d}}\left|a_{k j}\right| v(k-j)\left(\sup _{j \in \mathbb{Z}^{d}} \sum_{l \in \mathbb{Z}^{d}}\left|b_{j l}\right| v(j-l)\right) \\
& \leq\|A\|_{\mathcal{A}_{v}^{1}}\|B\|_{\mathcal{A}_{v}^{1}} \quad \forall k \in \mathbb{Z}^{d},
\end{aligned}
$$

and likewise for $\sup _{l} \sum_{k}(A B)_{k l}$.

(b) Since $v^{-1} \in \ell^{1}\left(\mathbb{Z}^{d}\right)$, we have $\sup _{k \in \mathbb{Z}^{d}} \sum_{l \in \mathbb{Z}^{d}}\left|a_{k l}\right| \leq C \sum_{l \in \mathbb{Z}^{d}} v(k-l)^{-1}=$ $C \sum_{l \in \mathbb{Z}^{d}} v(l)^{-1}<\infty$ and likewise for $\sup _{l} \sum_{k}$. Hence $\mathcal{A}_{v} \subseteq \mathcal{A}^{1}$. Now let $A, B \in \mathcal{A}_{v}$, 
then $\left|a_{k l}\right| \leq\|A\|_{\mathcal{A}_{v}} v(k-l)^{-1}$, and likewise for the entries of $B$. Combined with the subconvolutivity of $1 / v$, we obtain that

$$
\begin{aligned}
\left|(A B)_{k l}\right| & =\left|\sum_{j \in \mathbb{Z}^{d}} A_{k j} B_{j l}\right| \\
& \leq\|A\|_{\mathcal{A}_{v}}\|B\|_{\mathcal{A}_{v}} \sum_{j \in \mathbb{Z}^{d}} v(k-j)^{-1} v(j-l)^{-1} \\
& \leq\|A\|_{\mathcal{A}_{v}}\|B\|_{\mathcal{A}_{v}} C v(k-l)^{-1} \quad \forall k, l \in \mathbb{Z}^{d} .
\end{aligned}
$$

This means that $\|A B\|_{\mathcal{A}_{v}} \leq C\|A\|_{\mathcal{A}_{v}}\|B\|_{\mathcal{A}_{v}}$.

The algebra $\mathcal{A}_{v}$ is difficult to deal with, because banded matrices are not dense in $\mathcal{A}_{v}$ and because (8) is not a Banach algebra norm. (To obtain a Banach algebra, we would have to use the equivalent, but quite inconvenient, norm $\|A\|_{\mathcal{A}_{v}}^{\prime}=$ $\left.\sup _{D \in \mathcal{A}_{v},\|D\|_{\mathcal{A}_{v}=1}}\|A D\|_{\mathcal{A}_{v}}.\right)$ For the treatment of $\mathcal{A}_{v}$ we introduce a class of auxiliary algebras.

Definition 3. Let $s>0$, let $u$ be an admissible weight on $\mathbb{R}^{d}$ and set $v(x)=$ $u(x) \tau_{s}(x)=u(x)(1+|x|)^{s}$. We define $\mathcal{B}_{u, s}$ to be the Banach space $\mathcal{B}_{u, s}=\mathcal{A}_{u}^{1} \cap \mathcal{A}_{v}$ with norm

$$
\|A\|_{\mathcal{B}_{u, s}}=\kappa\|A\|_{\mathcal{A}_{u}^{1}}+\|A\|_{\mathcal{A}_{v}},
$$

where $\kappa \geq 1$ is the constant appearing in the subadditivity of $\tau_{s}$ in (4).

The following statement can be viewed as a generalization of Brandenburg's results in [6] from commutative convolution algebras to highly non-commutative algebras of matrices.

Lemma 2. (a) The class $\mathcal{B}_{u, s}$ with norm (9) is an involutive Banach algebra.

(b) If $s>d$, then $\mathcal{A}_{v} \subseteq \mathcal{A}_{u}^{1}$ and thus $\mathcal{B}_{u, s}=\mathcal{A}_{v}$ with equivalent norms.

Proof. (a) In addition to its submultiplicativity, the weight $v=u \tau_{s}$ satisfies the following inequality:

$$
\begin{aligned}
v(x+y) & =u(x+y) \tau_{s}(x+y) \\
& \leq \kappa u(x) u(y)\left(\tau_{s}(x)+\tau_{s}(y)\right) \\
& =\kappa(v(x) u(y)+u(x) v(y)) .
\end{aligned}
$$

If $A, B \in \mathcal{B}_{u, s}$, we obtain

$$
\begin{aligned}
\|A B\|_{\mathcal{A}_{v}}= & \sup _{k, m \in \mathbb{Z}^{d}}\left|\sum_{l \in \mathbb{Z}^{d}} a_{k l} b_{l m}\right| v(k-l+l-m) \\
\leq & \sup _{k, m \in \mathbb{Z}^{d}} \sum_{l \in \mathbb{Z}^{d}}\left|a_{k l}\right| \kappa v(k-l)\left|b_{l m}\right| u(l-m) \\
& \quad+\sup _{k, m \in \mathbb{Z}^{d}} \sum_{l \in \mathbb{Z}^{d}}\left|a_{k l}\right| \kappa u(k-l)\left|b_{l m}\right| v(l-m) \\
\leq & \kappa\|A\|_{\mathcal{A}_{v}} \sup _{m} \sum_{l}\left|b_{l m}\right| u(l-m)+\kappa\|B\|_{\mathcal{A}_{v}} \sup _{k} \sum_{l}\left|a_{k l}\right| u(k-l) \\
\leq & \kappa\left(\|A\|_{\mathcal{A}_{v}}\|B\|_{\mathcal{A}_{u}^{1}}+\|A\|_{\mathcal{A}_{u}^{1}}\|B\|_{\mathcal{A}_{v}}\right) .
\end{aligned}
$$


Using Lemma 11 and (9) we find that

$$
\begin{aligned}
\|A B\|_{\mathcal{B}_{u, s}} & =\kappa\|A B\|_{\mathcal{A}_{u}^{1}}+\|A B\|_{\mathcal{A}_{v}} \\
& \leq \kappa\|A\|_{\mathcal{A}_{u}^{1}}\|B\|_{\mathcal{A}_{u}^{1}}+\kappa\left(\|A\|_{\mathcal{A}_{v}}\|B\|_{\mathcal{A}_{u}^{1}}+\|A\|_{\mathcal{A}_{u}^{1}}\|B\|_{\mathcal{A}_{v}}\right) \\
& \leq\|A\|_{\mathcal{B}_{u, s}}\|B\|_{\mathcal{B}_{u, s}} .
\end{aligned}
$$

(b) If $s>d$, then $\sum_{k \in \mathbb{Z}^{d}} \tau_{s}^{-1}(k)=\sum_{k \in \mathbb{Z}^{d}}(1+|k|)^{-s}<\infty$ is summable, and thus $\sup _{k \in \mathbb{Z}^{d}} \sum_{l \in \mathbb{Z}^{d}}\left|a_{k l}\right| u(k-l) \tau_{s}(k-l) \tau_{s}(k-l)^{-1} \leq\|A\|_{\mathcal{A}_{v}} \sup _{k \in \mathbb{Z}^{d}} \sum_{l \in \mathbb{Z}^{d}} \tau_{s}(k-l)^{-1}=C\|A\|_{\mathcal{A}_{v}}$. Interchanging $k$ and $l$, we obtain $\|A\|_{\mathcal{A}_{u}^{1}} \leq C\|A\|_{\mathcal{A}_{v}}$, and so $\mathcal{A}_{v} \subseteq \mathcal{A}_{u}^{1}$ and $\mathcal{B}_{u, s}=$ $\mathcal{A}_{v}$.

Notation. We write $\sigma_{\mathcal{A}}(A)$ for the spectrum of a matrix $A$ in the algebra $\mathcal{A}$ and $\sigma(A)$ for the spectrum of $A$ as an operator acting on $\ell^{2}\left(\mathbb{Z}^{d}\right)$. The corresponding spectral radii are $\rho_{\mathcal{A}}(A)=\max \left\{|\lambda|: \lambda \in \sigma_{\mathcal{A}}(A)\right\}$ and $\rho(A)=\max \{|\lambda|: \lambda \in \sigma(A)\}$. By the spectral radius formula we have $\rho_{\mathcal{A}}(A)=\lim _{n \rightarrow \infty}\left\|A^{n}\right\|_{\mathcal{A}}^{1 / n}$. If $A=A^{*}$ is self-adjoint, then $\rho(A)=\|A\|_{o p}=\sup _{c \in \ell^{2}\left(\mathbb{Z}^{d}\right)}\|A c\|_{2} /\|c\|_{2}$.

2.3. Symmetric Banach algebras. A Banach algebra $\mathcal{A}$ is called symmetric if $\sigma_{\mathcal{A}}\left(A^{*} A\right) \subseteq[0, \infty)$ for all $A \in \mathcal{A}$. Equivalently, $\mathcal{A}$ is symmetric if and only if $A=A^{*} \in \mathcal{A}$ implies that $\sigma_{\mathcal{A}}(A) \subseteq \mathbb{R}$.

The standard example of a non-commutative symmetric algebra is the $C^{*}$-algebra of bounded operators $\mathcal{B}(\mathcal{H})$ on a Hilbert space $\mathcal{H}$ (as well as the norm-closed subalgebras of $\mathcal{B}(\mathcal{H})$ ).

A pair of nested Banach algebras $\mathcal{A} \subseteq \mathcal{B}$ is called a Wiener pair if $A \in \mathcal{A}$ and $A^{-1} \in \mathcal{B}$ implies $A^{-1} \in \mathcal{A}[16$. In the recent literature, one sometimes says that $\mathcal{A}$ is inverse closed in $\mathcal{B}[3,4]$ or that $\mathcal{A}$ is a spectral subalgebra of $\mathcal{B}[26,28$.

In the theory of Banach algebras the symmetry is interesting in its own right, because symmetric Banach algebras share many properties of $C^{*}$-algebras [27, 28]. While symmetry is defined intrinsically in terms of the Banach algebra itself, the concept of inverse-closedness describes a relation between two nested Banach algebras. Nevertheless, the two concepts are closely related, and almost always is the symmetry of a Banach algebra $\mathcal{A}$ proved by showing that it is inverse-closed in a $C^{*}$-algebra. Technically, this is accomplished by the following lemma of Hulanicki [23] (see also [15] for a corrected proof). We give a formulation that is most suitable for our purposes.

Lemma 3. Assume that $\mathcal{A}$ is an involutive Banach algebra with identity $\mathrm{I}_{\mathcal{H}}$ and contained in $\mathcal{B}(\mathcal{H})$. If for all self-adjoint $A=A^{*} \in \mathcal{A}$

$$
\rho_{\mathcal{A}}(A)=\rho(A)=\|A\|_{o p},
$$

then $\sigma_{\mathcal{A}}(A)=\sigma(A)$ for all $A \in \mathcal{A}$. Consequently, $\mathcal{A}$ and $\mathcal{B}(\mathcal{H})$ form a Wiener pair and $\mathcal{A}$ is a symmetric Banach algebra.

Note that if $\mathcal{A} \subseteq \mathcal{B}$ with a common unit, then $\sigma_{\mathcal{B}}(A) \subseteq \sigma_{\mathcal{A}}(A)$ and $\rho_{\mathcal{B}}(A) \leq$ $\rho_{\mathcal{A}}(A)$ for all $A \in \mathcal{A}$. So in order to apply Hulanicki's lemma and to show that $\mathcal{A} \subseteq \mathcal{B}(\mathcal{H})$ is symmetric, it suffices to show the reverse inequality

$$
\rho_{\mathcal{A}}(A) \leq \rho(a)=\|A\|_{o p}
$$

for all $A=A^{*} \in \mathcal{A}$. This is the strategy we will use in the sequel. 
To put the inverse-closedness in perspective, we briefly recall the Riesz functional calculus [31. Let $A \in \mathcal{B}$, let $U$ be an open neighborhood of (a connected component) of $\sigma_{\mathcal{B}}(A)$, let $\gamma \subseteq U \backslash \sigma_{\mathcal{B}}(A)$ be a "contour" of $\sigma_{\mathcal{B}}(A)$, and let $f$ be analytic on $U$. Then the $\mathcal{B}$-valued integral

$$
f(A)=\frac{1}{2 \pi i} \int_{\gamma} f(z)(A-z \mathrm{I})^{-1} d z
$$

is a well-defined element in $\mathcal{B}$, and the map $f \rightarrow f(A)$ is an algebra homomorphism. Now assume that $\mathcal{A}$ is inverse-closed in $\mathcal{B}$ and that $A \in \mathcal{A} \subseteq \mathcal{B}$. Since $\sigma_{\mathcal{A}}(A)=$ $\sigma_{\mathcal{B}}(A)$, the above integral is also well defined in $\mathcal{A}$. Thus we can formulate the following consequence of inverse-closedness.

Corollary 4. If $\mathcal{A}$ is inverse-closed in $\mathcal{B}$ with common identity, then Riesz functional calculi for $\mathcal{A}$ and $\mathcal{B}$ coincide.

2.4. Barnes' Lemma. The following statement shows that for weakly growing weights the algebras $\mathcal{A}_{v}^{1}$ are symmetric. Though important in its own right, it is only implicit in Barnes [3] (combine Lemma 4.6 with the main inequality in the proof of Theorem 4.7).

Lemma 5. Assume that $v(x)=(1+|x|)^{\delta}$ for $0<\delta \leq 1$. Then for all $A=A^{*} \in \mathcal{A}_{v}^{1}$ we have

$$
\rho_{\mathcal{A}_{v}^{1}}(A)=\rho_{\mathcal{A}^{1}}(A)=\|A\|_{o p} .
$$

In particular, $\mathcal{A}_{v}^{1}$ is symmetric.

It seems an open question as to whether $\mathcal{A}^{1}$ is also symmetric.

\section{SCHUR-TYPE CONDITIONS}

In this section we show that the matrix algebras $\mathcal{A}_{v}^{1}$ are inverse closed in $\mathcal{B}\left(\ell^{2}\left(\mathbb{Z}^{d}\right)\right)$ for a very general and useful class of weights, namely log-concave weights satisfying the GRS-condition and a very weak growth condition.

Our main theorem yields the symmetry of the algebras $\mathcal{A}_{v}^{1}$.

Theorem 6. Assume that $v$ is an admissible weight function satisfying the weak growth condition

$$
v(x) \geq C(1+|x|)^{\delta} \quad \text { for some } \delta, 0<\delta \leq 1 .
$$

Then

$$
\rho_{\mathcal{A}_{v}^{1}}(A)=\|A\|_{\text {op }} \quad \text { for all } A=A^{*} \in \mathcal{A}_{v}^{1} .
$$

Consequently, $\sigma_{\mathcal{A}_{v}^{1}}(A)=\sigma(A)$ for all $A \in \mathcal{A}_{v}^{1}$ and $\mathcal{A}_{v}^{1}$ is a symmetric Banach algebra.

We state the symmetry property and inverse closedness explicitly in the form that is needed in many applications.

Corollary 7. Assume that $v$ is an admissible weight function satisfying the condition $v(x) \geq C(1+|x|)^{\delta}$ for some $\delta>0$. If a matrix $A$ is invertible on $\ell^{2}\left(\mathbb{Z}^{d}\right)$ and satisfies the weighted Schur-type conditions

$$
\max \left\{\sup _{k} \sum_{l \in \mathbb{Z}^{d}}\left|a_{k l}\right| v(k-l), \sup _{l} \sum_{k \in \mathbb{Z}^{d}}\left|a_{k l}\right| v(k-l)\right\}<\infty,
$$


then the inverse matrix $A^{-1}=\left(b_{k l}\right)_{k, l \in \mathbb{Z}^{d}}$ satisfies the same conditions, i.e.,

$$
\max \left\{\sup _{k} \sum_{l \in \mathbb{Z}^{d}}\left|b_{k l}\right| v(k-l), \sup _{l} \sum_{k \in \mathbb{Z}^{d}}\left|b_{k l}\right| v(k-l)\right\}<\infty .
$$

If in addition $A$ acts as a positive operator on $\ell^{2}\left(\mathbb{Z}^{d}\right)$, then also the matrices corresponding to $A^{\alpha}$ for $\alpha \in \mathbb{R}$ are in $\mathcal{A}_{v}^{1}$.

For the proof of Theorem 6 we construct a sequence of auxiliary weights $v_{n}$ by using a technique developed in 29] and 20].

Lemma 8. For any unbounded admissible weight function $v$ there exists a sequence of admissible weights $v_{n}$ with the following properties:

(a) $v_{n+1} \leq v_{n} \leq v$ for all $n \in \mathbb{N}$,

(b) there exist $c_{n}>0$ such that $v \leq c_{n} v_{n}$, and

(c) $\lim _{n \rightarrow \infty} v_{n}=1$ uniformly on compact sets of $\mathbb{R}^{d}$.

Note that (a) implies that all $v_{n}$ satisfy the GRS-condition, and (a) and (b) imply that all the $v_{n}$ are equivalent. Consequently the algebras $\mathcal{A}_{v}^{1}$ and $\mathcal{A}_{v_{n}}^{1}$ coincide and have equivalent norms. In particular, we obtain that, for every $A \in \mathcal{A}_{v}^{1}$,

$$
\rho_{\mathcal{A}_{v}^{1}}(A)=\rho_{\mathcal{A}_{v_{n}}^{1}}(A) \quad \forall n \in \mathbb{N} .
$$

Proof. Since the concave function $\rho$ is strictly increasing (otherwise $v$ would be bounded), it has an inverse function $\rho^{-1}$.

Now set

$$
\gamma_{n}=\sup _{\mu \geq \rho^{-1}(n)} \frac{\rho(\mu)-n}{\mu}>0 .
$$

Since $\rho$ is continuous and $\lim _{\mu \rightarrow \infty} \frac{\rho(\mu)-n}{\mu}=0$ by the GRS condition (3), the supremum is assumed, and there exists a $\beta_{n} \geq \rho^{-1}(n)$ such that

$$
\gamma_{n}=\frac{\rho\left(\beta_{n}\right)-n}{\beta_{n}} .
$$

Now define the sequence of functions $\rho_{n}:[0, \infty) \rightarrow[0, \infty)$ as follows:

$$
\rho_{n}(\mu)= \begin{cases}\gamma_{n} \mu & \text { if } 0 \leq \mu \leq \beta_{n} \\ \rho(\mu)-n & \text { if } \mu \geq \beta_{n}\end{cases}
$$

The associated sequence of weights $v_{n}$ on $\mathbb{R}^{d}$ is then given by

$$
v_{n}(x)=e^{\rho_{n}(\|x\|)}, \quad x \in \mathbb{R}^{d} .
$$

We verify that this sequence of weights possesses the stated properties. By construction each $\rho_{n}$ is continuous and concave (it is a Legendre transform!) and satisfies $\rho_{n} \leq \rho$. Therefore the weights $v_{n}$ are admissible in the sense of Section 2.1.

By (18) $\gamma_{n+1} \leq \gamma_{n}$ and by the GRS condition $\lim _{n \rightarrow \infty} \gamma_{n}=0$, therefore $v_{n}(x)=$ $e^{\gamma_{n}\|x\|}$ for $\|x\| \leq \beta_{n} \rightarrow \infty$ converges to the constant function 1 uniformly on compact sets.

Further, since $v_{n}(x)=e^{-n} v(x)$ for $\|x\| \geq \beta_{n}$, we may set $c_{n}=\sup \left\{e^{n} ; \frac{v(x)}{v_{n}(x)},\|x\|\right.$ $\left.\leq \beta_{n}\right\}$ and obtain that $v(x) \leq c_{n} v_{n}(x)$.

The monotonicity $v_{n+1} \leq v_{n}$ is clear for $\|x\| \geq \beta_{n}$, because $\rho_{n+1}(\mu)=\rho_{n}(\mu)-1$ for $\mu \geq \beta_{n}$. On the complement we observe that $\rho_{n+1}(0)=\rho_{n}(0)$ and $\rho_{n+1}\left(\beta_{n+1}\right)<$ 


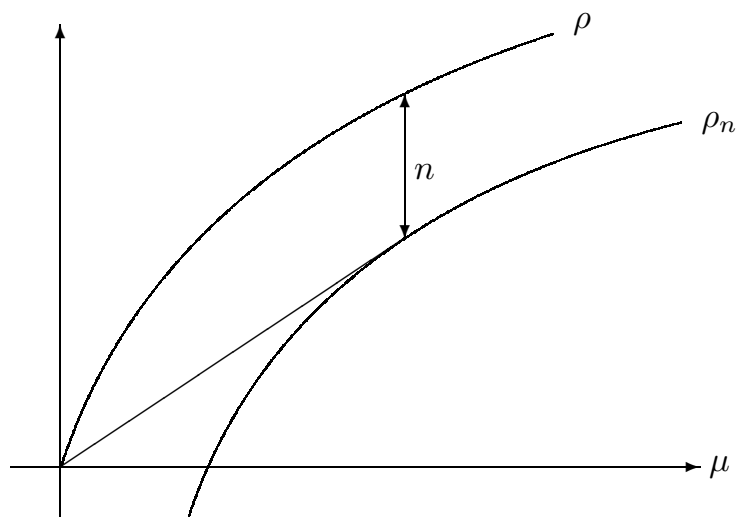

Figure 1. Construction of the modified weights (20).

$\rho_{n}\left(\beta_{n+1}\right)$. Therefore the graph of the linear function $\rho_{n+1}$ lies under the concave function $\rho_{n}$ on $\left[0, \beta_{n+1}\right]$, and so $v_{n+1} \leq v_{n}$ on all of $\mathbb{R}^{d}$.

Lemma 9. Under the hypotheses of Theorem 6 and with $v_{n}$ as in Lemma 8, the following identities hold for every $A=A^{*} \in \mathcal{A}_{v}^{1}$ :

$$
\begin{gathered}
\lim _{n \rightarrow \infty}\|A\|_{\mathcal{A}_{v_{n}}^{1}}=\|A\|_{\mathcal{A}^{1}}, \text { and } \\
\rho_{\mathcal{A}_{v}^{1}}(A)=\rho_{\mathcal{A}^{1}}(A)=\|A\|_{o p} .
\end{gathered}
$$

Proof. (a) Let $\epsilon>0$. For self-adjoint $A \in \mathcal{A}_{v}^{1}$ we have

$$
\|A\|_{\mathcal{A}_{v_{n}}^{1}}=\sup _{k \in \mathbb{Z}^{d}} \sum_{l \in \mathbb{Z}^{d}}\left|a_{k l}\right| v_{n}(k-l) .
$$

Since by construction of $v_{n}$ we have $v_{n}(x)=e^{-n} v(x)$ for $\|x\| \geq \beta_{n}$, there is some $n_{0}=n_{0}(\epsilon) \in \mathbb{N}$ such that

$$
\sup _{k \in \mathbb{Z}^{d}} \sum_{l \in \mathbb{Z}^{d}:\|k-l\| \geq \beta_{n_{0}}}\left|a_{k l}\right| v_{n_{0}}(k-l) \leq e^{-n_{0}}\|A\|_{\mathcal{A}_{v}^{1}}<\epsilon .
$$

By monotonicity $v_{n+1} \leq v_{n} \leq v$ for all $n$ we therefore obtain that for all $n \geq n_{0}$

$$
\sup _{k \in \mathbb{Z}^{d}} \sum_{l \in \mathbb{Z}^{d}:\|k-l\| \geq \beta_{n_{0}}}\left|a_{k l}\right| v_{n}(k-l)<\epsilon .
$$

If $\|x\| \leq \beta_{n_{0}}$, then $v_{n}$ converges to 1 uniformly, so for $n \geq n_{1}=n_{1}(\epsilon)$ we have that

$$
\sup _{k \in \mathbb{Z}^{d}} \sum_{l \in \mathbb{Z}^{d}:\|k-l\| \leq \beta_{n_{0}}}\left|a_{k l}\right| v_{n}(k-l) \leq(1+\epsilon) \sup _{k \in \mathbb{Z}^{d}} \sum_{l \in \mathbb{Z}^{d}}\left|a_{k l}\right| .
$$

Combining these estimates we obtain that for $n \geq \max \left(n_{0}, n_{1}\right)$

$$
\|A\|_{\mathcal{A}_{v_{n}}^{1}} \leq \epsilon+(1+\epsilon)\|A\|_{\mathcal{A}^{1}} .
$$

We conclude that

$$
\lim _{n \rightarrow \infty}\|A\|_{\mathcal{A}_{v_{n}}^{1}} \leq\|A\|_{\mathcal{A}^{1}} \quad \forall A \in \mathcal{A}_{v}^{1} .
$$

The reverse inequality is obvious, since $v_{n} \geq 1$ for all $n$. 
(b) Using step (a) and the equivalence of the weights $v$ and $v_{n}$, we then have

$$
\rho_{\mathcal{A}_{v}^{1}}(A)^{k}=\rho_{\mathcal{A}_{v}^{1}}\left(A^{k}\right)=\rho_{\mathcal{A}_{v_{n}}^{1}}\left(A^{k}\right) \leq\left\|A^{k}\right\|_{\mathcal{A}_{v_{n}}^{1}} \quad \forall n \in \mathbb{N} .
$$

Consequently,

$$
\rho_{\mathcal{A}_{v}^{1}}(A)^{k} \leq \lim _{n \rightarrow \infty}\left\|A^{k}\right\|_{\mathcal{A}_{v_{n}}^{1}}=\left\|A^{k}\right\|_{\mathcal{A}^{1}} \quad \forall k \in \mathbb{N},
$$

and so by taking $k$-th roots we have

$$
\rho_{\mathcal{A}_{v}^{1}}(A) \leq \lim _{k \rightarrow \infty}\left\|A^{k}\right\|_{\mathcal{A}^{1}}^{1 / k}=\rho_{\mathcal{A}^{1}}(A) .
$$

Since $v(x) \geq C(1+|x|)^{\delta}=\tau_{\delta}(x)$ and $\delta>0$, we have the inclusion $\mathcal{A}_{v}^{1} \subseteq \mathcal{A}_{\tau_{\delta}}^{1}$, and so Barnes's Lemma (Lemma 5) shows that $\rho_{\mathcal{A}^{1}}(A)=\|A\|_{o p}$. Consequently, $\rho_{\mathcal{A}_{v}^{1}}(A)=\|A\|_{o p}$, as desired.

Proof of Theorem 6 and Corollary 7 . We combine Hulanicki's Lemma 3 with the identity for spectral radii (23) of Lemma 9 and conclude the equality of the spectra $\sigma(A)=\sigma_{\mathcal{A}_{v}^{1}}(A)$ for all $A \in \mathcal{A}_{v}^{1}$. This implies that $\mathcal{A}_{v}^{1}$ is a symmetric Banach algebra.

In particular, if $A \in \mathcal{A}_{v}^{1}$ is invertible on $\ell^{2}\left(\mathbb{Z}^{d}\right)$, then $A$ is also invertible as an element of $\mathcal{A}_{v}^{1}$.

Now assume that $A \in \mathcal{A}_{v}^{1}$ is invertible and induces a positive operator on $\ell^{2}\left(\mathbb{Z}^{d}\right)$. Then $\sigma(A) \subseteq\left[\delta,\|A\|_{o p}\right]$ for some $\delta>0$, and thus $\sigma_{\mathcal{A}_{v}^{1}}(A) \subseteq\left[\delta,\|A\|_{o p}\right]$. By the Riesz functional calculus (Corollary 4 or [31, Thm. 3.10]) all powers $A^{\alpha}$ are in $\mathcal{A}_{v}^{1}$.

Remarks. 1. Note the Lemma 8 is false when $v$ does not satisfy the GRS-condition. For instance, if $v$ is an exponential weight, where $v(x)=e^{\alpha|x|}$ for some $\alpha>0$, then Lemma 8 fails.

Theorem [ 6 is sharp in dimension $d=1$ as is shown by adaption of an example in [16. Assume that $\lim _{n \rightarrow \infty} v\left(n k_{0}\right)^{1 / n}=\beta>1$ for some $k_{0} \in \mathbb{N}$. Then since $v$ is submultiplicative, we have

$$
\lim _{n \rightarrow \infty} v(n)^{1 / n}=\inf _{n \in \mathbb{N}} v(n)^{1 / n}=\beta^{1 / k_{0}}=e^{\alpha}
$$

for some $\alpha>0$.

Let $h$ be the coefficients of the trigonometric polynomial $\hat{h}(\omega)=e^{2 \pi i \omega}-e^{-\delta} i$ and consider the associated convolution operator $A c=h * c$ on $\ell^{2}(\mathbb{Z})$. Its matrix has the entries $A_{k l}=h_{k-l}$, so $A$ is in fact a banded matrix and $\|A\|_{\mathcal{A}_{v}^{1}}=\|h\|_{\ell_{v}^{1}}<\infty$. Clearly $\hat{h}(\omega) \neq 0, \forall \omega$, and by explicit computation we have $\hat{g}(\omega)=1 / \hat{h}(\omega)=$ $\sum_{n=0}^{\infty} e^{-\delta n} i^{n} e^{-2 \pi i(n+1) \omega}$. Therefore $A$ is invertible with inverse $A^{-1} c=g * c$. Choosing $\delta<\alpha$, then we conclude from (24) that $\left\|A^{-1}\right\|_{\mathcal{A}_{v}^{1}}=\sum_{n=0}^{\infty} e^{-\delta n} v(n)=\infty$. For a weight $v$ violating the GRS-condition, Theorem [6 is false.

2. In some applications more general index sets are needed. The proof of Theorem 6 carries over to arbitrary countable index sets $\mathcal{N}$ endowed with a non-trivial metric $d$. To apply Lemma 5, we have to assume that the volume of balls $B(x, r)$ grows polynomially in the radius $r$ and independently of $x \in \mathcal{N}$ (see condition (4.1) in [3]). For further reference we formulate a version of Corollary 7 explicitly: Assume that $\rho:[0, \infty) \rightarrow[0, \infty)$ is a continuous, concave function satisfying $\rho(0)=0, \rho(t) \geq C+\delta \log (1+t)$ for some $C \geq 0$ and $\delta \in(0,1]$ and $\lim _{t \rightarrow \infty} \frac{\rho(t)}{t}=0$. 
Set $v(m, n)=e^{\rho(d(m, n))}$ for $m, n \in \mathcal{N}$. If a matrix $A=\left(a_{m n}\right)_{m, n \in \mathcal{N}}$ is invertible on $\ell^{2}(\mathcal{N})$ and satisfies the estimates

$$
\max \left\{\sup _{m \in \mathcal{N}} \sum_{n \in \mathcal{N}}\left|a_{m n}\right| v(m, n), \sup _{n \in \mathcal{N}} \sum_{m \in \mathcal{N}}\left|a_{m n}\right| v(m, n)\right\}<\infty,
$$

then the inverse matrix $A^{-1}=\left(b_{m n}\right)_{m, n \in \mathcal{N}}$ satisfies the same conditions

$$
\max \left\{\sup _{m \in \mathcal{N}} \sum_{n \in \mathcal{N}}\left|b_{m n}\right| v(m, n), \sup _{n \in \mathcal{N}} \sum_{m \in \mathcal{N}}\left|b_{m n}\right| v(m, n)\right\}<\infty .
$$

\section{Off-Diagonal DECAY OF INVERSE MATRICES}

We next consider the matrix algebras $\mathcal{B}_{u, s}$ and $\mathcal{A}_{v}$. The following statement is a consequence of Theorem 6 .

Theorem 10. Assume that $u$ is an admissible weight, $\delta, s>0, u \geq \tau_{\delta}$, and $v=u \tau_{s}$. Then

$$
\rho_{\mathcal{B}_{u, s}}(A)=\|A\|_{o p} \quad \text { for all } A=A^{*} \in \mathcal{B}_{u, s} .
$$

Consequently, $\sigma_{\mathcal{B}_{u, s}}(A)=\sigma(A)$ and $\mathcal{B}_{u, s}$ is a symmetric Banach algebra.

Proof. To establish the required identity of spectral radii, we follow [6].

Assume that $A \in \mathcal{B}_{u, s} \subseteq \mathcal{A}_{v}$; then the important inequality (10) (used for establishing that $\mathcal{B}_{u, s}$ is a Banach algebra) implies that

$$
\left\|A^{2 n}\right\|_{\mathcal{A}_{v}} \leq 2 \kappa\left\|A^{n}\right\|_{\mathcal{A}_{u}^{1}}\left\|A^{n}\right\|_{\mathcal{A}_{v}} \quad \forall n \geq 1 .
$$

Consequently

$$
\begin{aligned}
\left\|A^{2 n}\right\|_{\mathcal{B}_{u, s}} & =\kappa\left\|A^{2 n}\right\|_{\mathcal{A}_{u}^{1}}+\left\|A^{2 n}\right\|_{\mathcal{A}_{v}} \\
& \leq \kappa\left\|A^{n}\right\|_{\mathcal{A}_{u}^{1}}^{2}+2 \kappa\left\|A^{n}\right\|_{\mathcal{A}_{u}^{1}}\left\|A^{n}\right\|_{\mathcal{A}_{v}} \\
& \leq 2 \kappa\left\|A^{n}\right\|_{\mathcal{A}_{u}^{1}}\left(\kappa\left\|A^{n}\right\|_{\mathcal{A}_{u}^{1}}+\left\|A^{n}\right\|_{\mathcal{A}_{v}}\right) \\
& =2 \kappa\left\|A^{n}\right\|_{\mathcal{A}_{u}^{1}}\left\|A^{n}\right\|_{\mathcal{B}_{u, s}} .
\end{aligned}
$$

By taking roots we obtain

$$
\begin{aligned}
\rho_{\mathcal{B}_{u, s}}(A) & =\lim _{n \rightarrow \infty}\left\|A^{2 n}\right\|_{\mathcal{B}_{u, s}}^{\frac{1}{2 n}} \\
& \leq \lim _{n \rightarrow \infty}(2 \kappa)^{\frac{1}{2 n}}\left\|A^{n}\right\|_{\mathcal{A}_{u}^{1}}^{\frac{1}{2 n}}\left\|A^{n}\right\|_{\mathcal{B}_{u, s}}^{\frac{1}{2 n}} \\
& =\rho_{\mathcal{A}_{u}^{1}}(A)^{1 / 2} \rho_{\mathcal{B}_{u, s}}(A)^{1 / 2} .
\end{aligned}
$$

This implies that

$$
\rho_{\mathcal{B}_{u, s}}(A) \leq \rho_{\mathcal{A}_{u}^{1}}(A) .
$$

Since $\mathcal{A}_{u}^{1} \subseteq \mathcal{A}_{\tau_{\delta}}^{1}$, we can apply Theorem 6 and obtain

$$
\rho_{\mathcal{B}_{u, s}}(A) \leq \rho_{\mathcal{A}_{u}^{1}}(A)=\rho_{\mathcal{A}^{1}}(A)=\|A\|_{o p} \quad \forall A=A^{*} \in \mathcal{B}_{u, s} .
$$

Consequently we have derived the identity

$$
\rho_{\mathcal{B}_{u, s}}(A)=\|A\|_{o p} \quad \forall A=A^{*} \in \mathcal{B}_{u, s} .
$$

The remainder of the proof is as in the proof of Theorem [6 we use Hulanicki's Lemma 3 to conclude that $\sigma_{\mathcal{B}_{u, s}}(A)=\sigma(A)$ for all $A \in \mathcal{B}_{u, s}$, and we are done.

For $s>d$ we have $\mathcal{B}_{u, s}=\mathcal{A}_{v}$, and so we obtain the following result on the inversion of matrices with a given off-diagonal decay. 
Corollary 11. Assume that $u$ is an admissible weight, $s>d$, and $v=u \tau_{s}$. If $A$ is invertible on $\ell^{2}\left(\mathbb{Z}^{d}\right)$ and satisfies the off-diagonal condition

$$
\left|a_{k l}\right| \leq C v(k-l)^{-1},
$$

then the inverse matrix $B=A^{-1}$ satisfies the condition

$$
\left|b_{k l}\right| \leq C^{\prime} v(k-l)^{-1} \text {. }
$$

If in addition $A$ is positive and invertible, then the matrices $B=A^{\alpha}$ for $\alpha \in \mathbb{R}$ also satisfy a decay condition like (27).

Proof. For $s>d$ the algebras $\mathcal{B}_{u, s}$ and $\mathcal{A}_{v}$ coincide by Lemma 2 Thus the statement follows from Theorem 10 .

Remarks. 1. By choosing $v(x)=\tau_{d+\delta}$ for $\delta>0$, we recover Jaffard's Theorem 24] with an entirely different proof.

2. For completeness we formulate a version of Corollary 11 for arbitrary index sets $\mathcal{N}$ with a metric $d$. Assume that $\rho:[0, \infty) \rightarrow[0, \infty)$ is continuous, concave, $\rho(0)=0$ and $\lim _{t \rightarrow \infty} \frac{\rho(t)}{t}=0$. Set $v(m, n)=e^{\rho(d(m, n))}(1+d(m, n))^{s}$ for $m, n \in \mathcal{N}$, where $s$ is chosen such that $\sup _{m \in \mathcal{N}} \sum_{n \in \mathcal{N}}(1+d(m, n))^{-s}<\infty$. If a matrix $A=\left(a_{m, n}\right)_{m, n \in \mathcal{N}}$ is invertible on $\ell^{2}(\mathcal{N})$ and satisfies the estimates

$$
\sup _{m, n \in \mathcal{N}}\left|a_{m n}\right| v(m, n)<\infty,
$$

then the inverse matrix $A^{-1}=\left(b_{m n}\right)_{m, n \in \mathcal{N}}$ also satisfies

$$
\sup _{m, n \in \mathcal{N}}\left|b_{m n}\right| v(m, n)<\infty \text {. }
$$

\section{Applichtions}

In this section we present two applications of the previous theorems. In fact, it is exactly these topics that have motivated us to seek refinements of Jaffard's Theorem. Since the context and the connections to related areas are well documented in the cited literature and would only be distractive, we will focus on the mathematical key points. In the first problem (twisted convolution) the application of Theorem 6 yields a significantly shorter proof than the original one. In the second problem our treatment sheds new light on the role of matrix algebras in frame theory.

\subsection{Wiener's Lemma for twisted convolution.}

Definition 4. Given $\theta>0$, the twisted convolution of two sequences $\mathbf{a}=\left(a_{k l}\right)_{k, l \in \mathbb{Z}^{d}}$ and $\mathbf{b}=\left(b_{k l}\right)_{k, l \in \mathbb{Z}^{d}}$ on $\mathbb{Z}^{2 d}$ (with finite support) is defined to be

$$
\left(\mathbf{a} \natural_{\theta} \mathbf{b}\right)(m, n)=\sum_{k, l \in \mathbb{Z}^{d}} a_{k l} b_{m-k, n-l} e^{2 \pi i \theta(m-k) \cdot l}=\sum_{k, l \in \mathbb{Z}^{d}} a_{m-k, n-l} e^{2 \pi i \theta k \cdot(n-l)} b_{k l} .
$$

Since

$$
\left\|\mathbf{a} \natural_{\theta} \mathbf{b}\right\|_{p} \leq\||\mathbf{a}| *|\mathbf{b}|\|_{p} \leq\|\mathbf{a}\|_{1}\|\mathbf{b}\|_{p},
$$

the twisted convolution operator $T_{\mathbf{a}} \mathbf{c}=\mathbf{a} \natural_{\theta} \mathbf{c}$ is bounded on $\ell^{p}\left(\mathbb{Z}^{2 d}\right)$ for any $\mathbf{a} \in$ $\ell^{1}\left(\mathbb{Z}^{2 d}\right)$. 
We next apply Theorem 6 and Corollary 7 to this special class of operators and give a new and significantly shorter proof of Wiener's Lemma for twisted convolution in [20].

Theorem 12. Assume that (a) $\mathbf{a} \in \ell_{v}^{1}\left(\mathbb{Z}^{2 d}\right)$ for an admissible weight satisfying the GRS-condition (3) and (13) and that (b) the (twisted) convolution operator $T_{\mathbf{a}}$ is invertible on $\ell^{2}\left(\mathbb{Z}^{2 d}\right)$.

Then $\mathbf{a}$ is invertible in $\ell_{v}^{1}\left(\mathbb{Z}^{2 d}\right)$ and so $T_{\mathbf{a}}^{-1}=T_{\mathbf{b}}$ for some $\mathbf{b} \in \ell_{v}^{1}\left(\mathbb{Z}^{2 d}\right)$. Consequently $T_{\mathbf{a}}$ is invertible simultaneously on all $\ell_{v}^{p}\left(\mathbb{Z}^{2 d}\right)$ for $1 \leq p \leq \infty$.

Proof. By (29) the matrix $A$ associated to $T_{\mathbf{a}}$ has the entries

$$
A_{(k, l),(m, n)}=a_{m-k, n-l} e^{2 \pi i \theta k \cdot(n-l)} .
$$

Consequently for $\mathbf{a} \in \ell_{v}^{1}\left(\mathbb{Z}^{2 d}\right)$ we have

$$
\sup _{(k, l) \in \mathbb{Z}^{2 d}} \sum_{(m, n) \in \mathbb{Z}^{2 d}}\left|A_{(k, l),(m, n)}\right| v(k-m, l-n)=\|\mathbf{a}\|_{\ell_{v}^{1}}<\infty,
$$

and likewise with indices interchanged. Thus $\|A\|_{\mathcal{A}_{v}^{1}}=\|\mathbf{a}\|_{\ell_{v}^{1}}$ and $A \in \mathcal{A}_{v}^{1}$. Now by Theorem $\left[\right.$ we also have $B:=A^{-1} \in \mathcal{A}_{v}^{1}$. It is left to show that $B$ corresponds to a (twisted) convolution operator $T_{\mathbf{b}}$. So let $\mathbf{b} \in \ell^{2}\left(\mathbb{Z}^{2 d}\right)$ be the solution of $T_{\mathbf{a}} \mathbf{b}=\delta$ with $\delta(0)=1$ and $\delta(k)=0$ for $k \in \mathbb{Z}^{2 d} \backslash\{0\}$. The (twisted) convolution operator $T_{\mathbf{b}}$ is certainly defined on the dense subspace $\ell^{0}\left(\mathbb{Z}^{2 d}\right)=\{\mathbf{c}: \operatorname{supp} \mathbf{c}$ is finite $\}$, and by a version of (30) it maps $\ell^{0}\left(\mathbb{Z}^{2 d}\right)$ into $\ell^{2}\left(\mathbb{Z}^{2 d}\right)$. Then for all $\mathbf{c} \in \ell^{0}\left(\mathbb{Z}^{2 d}\right)$

$$
T_{\mathbf{a}}\left(T_{\mathbf{b}}-B\right) \mathbf{c}=\mathbf{a} \natural_{\theta}\left(\mathbf{b} \natural_{\theta} \mathbf{c}\right)-T_{\mathbf{a}} T_{\mathbf{a}}^{-1} \mathbf{c}=\mathbf{c}-\mathbf{c}=0 .
$$

Since we have $T_{\mathbf{b}}=B$ on the dense subspace $\ell^{0}\left(\mathbb{Z}^{2 d}\right)$, the matrix of $T_{\mathbf{b}}$ coincides with $B$, and so (31) implies that $\mathbf{b} \in \ell_{v}^{1}\left(\mathbb{Z}^{2 d}\right)$.

Remark. 1. The original proof in 20 is more complicated and uses the special structure of the twisted convolution and its relation to the representation theory of certain groups of Heisenberg-type and to the rotation algebras in operator theory.

2. Strictly speaking, the unweighted case does not follow from Theorem 6 because of the additional condition (13). In this case, one may use a little known lemma of Sjöstrand [32] which states that a certain subalgebra of $\mathcal{A}^{1}$ is inverse-closed in $\mathcal{B}\left(\ell^{2}\right)$.

3. Theorem 12 is the main ingredient in the construction of Gabor frames with good time-frequency concentration (as measured by the decay of the short-time Fourier transform); see [20, 14, 34] for the necessary background on time-frequency analysis and signal analysis.

5.2. Localization of frames. A frame in a Hilbert space $\mathcal{H}$ is an overcomplete set that yields stable series expansions similar to orthonormal expansions, but with more flexibility. While the concept of a frame is a pure Hilbert space concept, most (mathematical and real) applications make use of additional features of frames, namely structure (Gabor frames, frames of reproducing kernels, wavelet frames, etc.) and localization. While general frames and structured frames are well understood and are the subject of many treatises $[7,6,11,17,22$, the notion of localized frames is a new idea [18, 19, 2] and far from being fully explored. The concept of localization makes frame expansions meaningful for other Banach spaces besides $\mathcal{H}$ [19] and plays a key role in the characterization of certain Banach spaces by means of frames and in the investigations of sparse representations with frames [19, 10]. 
Here we introduce a new version of localized frames that involves matrix algebras and highlights the role of their Banach algebra properties. We will provide a refinement of the concept of localized frames that bridges the gap between polynomial localization and exponential localization defined in [19].

A set $\mathcal{E}=\left\{e_{x}: x \in \mathcal{X}\right\}$ is a frame for a Hilbert space $\mathcal{H}$ if the associated frame operator $S f:=\sum_{x \in \mathcal{X}}\left\langle f, e_{x}\right\rangle e_{x}$ is bounded on $\mathcal{H}$ and has a bounded inverse. Here $\mathcal{X} \subseteq \mathbb{R}^{d}$ is a relatively separated set; this means that

$$
\sup _{k \in \mathbb{Z}^{d}} \operatorname{card}\left\{x \in \mathcal{X}: x \in k+[0,1]^{d}\right\}=\nu<\infty .
$$

(In a function space we think of the subscript $x$ as the center of the "essential support" of $e_{x}$.) Since $S$ is invertible, every $f \in \mathcal{H}$ has an expansion of the form $f=\sum_{x \in \mathcal{X}}\left\langle f, e_{x}\right\rangle S^{-1} e_{x}=\sum_{x \in \mathcal{X}}\left\langle f, S^{-1} e_{x}\right\rangle e_{x}$, where $\widetilde{\mathcal{E}}=\left\{S^{-1} e_{x}: x \in \mathcal{X}\right\}$ is the dual frame.

Let $\mathcal{A}$ be an involutive Banach algebra of infinite matrices contained in $\mathcal{B}\left(\ell^{2}\left(\mathbb{Z}^{d}\right)\right)$ with the following properties: (a) $\mathcal{A}$ is inverse-closed in $\mathcal{B}\left(\ell^{2}\left(\mathbb{Z}^{d}\right)\right)$, and (b) $\mathcal{A}$ is solid, i.e., if $A=\left(a_{k l}\right) \in \mathcal{A}$ and $\left|b_{k l}\right| \leq\left|a_{k l}\right|$, then the matrix $B=\left(b_{k l}\right)$ is also in $\mathcal{A}$. The matrix algebras $\mathcal{A}_{v}^{1}, \mathcal{B}_{u, s}$ and $\mathcal{A}_{v}$ are solid by definition of the norm and inverse-closed by Theorems 6 and 10 .

In the following we compare a frame $\mathcal{E}$ to a Riesz basis. Let $\left\{g_{k}: k \in \mathbb{Z}^{d}\right\}$ be a Riesz basis of $\mathcal{H}$ with dual basis $\left\{\widetilde{g}_{l}: l \in \mathbb{Z}^{d}\right\}$.

Since the index set is relatively separated, we can majorize $\left\langle e_{x}, g_{l}\right\rangle$ for $x \in$ $k+[0,1]^{d}$ by

$$
\left|\left\langle e_{x}, g_{l}\right\rangle\right| \leq \sum_{x \in \mathcal{X} \cap\left(k+[0,1]^{d}\right)}\left|\left\langle e_{x}, g_{l}\right\rangle\right| \leq \nu \max _{x \in \mathcal{X} \cap\left(k+[0,1]^{d}\right)}\left|\left\langle e_{x}, g_{l}\right\rangle\right|:=b_{k l} .
$$

We set $b_{k l}=0$ if $\mathcal{X} \cap\left(k+[0,1]^{d}\right)=\emptyset$. Likewise we define a matrix $C$ by the entries $c_{k l}:=\nu \max _{x \in \mathcal{X} \cap\left(k+[0,1]^{d}\right)}\left|\left\langle e_{x}, \widetilde{g}_{l}\right\rangle\right|$.

Definition 5. A frame $\mathcal{E}$ for $\mathcal{H}$ is called $\mathcal{A}$-localized with respect to the Riesz basis $\left\{g_{k}: k \in \mathbb{Z}^{d}\right\}$, if both $B \in \mathcal{A}$ and $C \in \mathcal{A}$.

In particular, if $\mathcal{A}=\mathcal{A}_{v}$ for an admissible weight $v$ on $\mathbb{R}^{d}$, then Definition 5 implies that that $\mathcal{E}$ is $\mathcal{A}_{v}$-localized if

$$
\max \left\{\left|\left\langle e_{x}, g_{k}\right\rangle\right|,\left|\left\langle e_{x}, \widetilde{g_{k}}\right\rangle\right|\right\} \leq C v(x-k)^{-1}, \quad x \in \mathcal{X}, k \in \mathbb{Z}^{d} .
$$

For $v(x)=(1+|x|)^{s}$ we recover the notion of polynomial localization of [19].

The main theorem of [19] can be generalized as follows:

Theorem 13. If $\mathcal{E}$ is an $\mathcal{A}$-localized frame for $\mathcal{H}$, then its dual frame $\widetilde{\mathcal{E}}$ is also $\mathcal{A}$-localized.

Proof. The proof is identical to the proof of [19, Thm. 3.5b]. We just substitute Jaffard's Theorem by the property that $\mathcal{A}$ is inverse-closed in $\mathcal{B}\left(\ell^{2}\left(\mathbb{Z}^{2 d}\right)\right)$. To give the reader an idea how it works, we indicate the main lines of the proof. We keep the notation of $[19$.

Step 1. Let $(\Gamma f)(k)=\left\langle f, \widetilde{g_{k}}\right\rangle$. Since $\left\{g_{k}: k \in \mathbb{Z}^{d}\right\}$ is a Riesz basis of $\mathcal{H}$, $\Gamma$ is an isomorphism from $\mathcal{H}$ onto $\ell^{2}\left(\mathbb{Z}^{d}\right)$ with inverse $\Gamma^{-1} \mathbf{c}=\sum_{k \in \mathbb{Z}^{d}} c_{k} g_{k}$ for $\mathbf{c}=\left(c_{k}\right) \in \ell^{2}\left(\mathbb{Z}^{d}\right)$. Then the frame operator $S$ can be factored as $S=\Gamma^{-1} T \Gamma$, where $T$ is the matrix of $S$ with respect to the given Riesz basis. It has the entries

$$
T_{k l}=\left\langle S g_{l}, \widetilde{g_{k}}\right\rangle=\sum_{x \in \mathcal{X}}\left\langle g_{l}, e_{x}\right\rangle\left\langle e_{x}, \widetilde{g_{k}}\right\rangle .
$$


Note that $T$ is invertible on $\ell^{2}\left(\mathbb{Z}^{d}\right)$ if and only if $S$ is invertible on $\mathcal{H}$.

Step 2. Estimating the entries of $T$,

$$
\begin{aligned}
\left|T_{k l}\right| & \leq \sum_{m \in \mathbb{Z}^{d}} \sum_{x \in \mathcal{X} \cap\left(m+[0,1]^{d}\right)}\left|\left\langle g_{l}, e_{x}\right\rangle\left\langle e_{x}, \widetilde{g_{k}}\right\rangle\right| \\
& \leq \sum_{m \in \mathbb{Z}^{d}} b_{m l} c_{m k}=\left(C^{*} B\right)_{k l} .
\end{aligned}
$$

By hypothesis $B, C \in \mathcal{A}$, therefore $T \in \mathcal{A}$ as well.

Step 3. Since $T \in \mathcal{A}$ and $T$ is invertible on $\ell^{2}\left(\mathbb{Z}^{2 d}\right)$, we conclude that $T^{-1} \in \mathcal{A}$. (This is where Theorems 6 and 10 come in.) Let $T^{-1}=\left(u_{k l}\right)$; then the matrix $U=\left(\left|u_{k l}\right|\right)$ is also in $\mathcal{A}$.

Step 4. To show that $\widetilde{\mathcal{E}}$ is $\mathcal{A}$-localized, we must check the size of $\left\langle S^{-1} e_{x}, g_{l}\right\rangle$ and of $\left\langle S^{-1} e_{x}, \widetilde{g}_{l}\right\rangle$. We use that $S^{-1}=\Gamma^{-1} T^{-1} \Gamma$ and obtain that

$$
\begin{aligned}
\widetilde{c}_{k l} & =\nu \max _{x \in \mathcal{X} \cap\left(k+[0,1]^{d}\right)}\left|\left\langle S^{-1} e_{x}, \widetilde{g_{l}}\right\rangle\right| \\
& =\nu \max _{x \in \mathcal{X} \cap\left(k+[0,1]^{d}\right)}\left|\left(\Gamma S^{-1} e_{x}\right)(l)\right| \\
& =\nu \max _{x \in \mathcal{X} \cap\left(k+[0,1]^{d}\right)}\left|\sum_{m \in \mathbb{Z}^{d}}\left(T^{-1}\right)_{l m}\left\langle e_{x}, \widetilde{g_{m}}\right\rangle\right| \\
& \leq \nu \sum_{m \in \mathbb{Z}^{d}}\left|u_{l m}\right| \max _{x \in \mathcal{X} \cap\left(k+[0,1]^{d}\right)}\left|\left\langle e_{x}, \widetilde{g_{m}}\right\rangle\right| \\
& =\sum_{m \in \mathbb{Z}^{d}}\left|u_{l m}\right| c_{k m}=\left(C U^{*}\right)_{k l} .
\end{aligned}
$$

Since $C, U \in \mathcal{A}$ and $\mathcal{A}$ is an algebra, we find that $\tilde{C} \in \mathcal{A}$. By interchanging the role of $g_{k}$ and $\tilde{g_{k}}$, we obtain that the matrix $\tilde{B}$ with entries

$$
\tilde{b}_{k l}=\max _{x \in \mathcal{X} \cap\left(k+[0,1]^{d}\right)}\left|\left\langle S^{-1} e_{x}, g_{l}\right\rangle\right|
$$

is also in $\mathcal{A}$. Thus $\widetilde{\mathcal{E}}$ is $\mathcal{A}$-localized.

For examples on how Theorem 13 can be applied we refer to the sections on sampling theorems and Gabor frames in 19. These sections could be rewritten for $\mathcal{A}$-localized frames instead of polynomially localized frames and yield stronger conclusions.

\section{ACKNOWLEDGEMENT}

Research on this paper was done when the second author visited the University of Connecticut. He would like to express his appreciation for the hospitality of the host institution. We also thank Tobias Werther for offering help in plotting Figure 1 and Scott Beaver for carefully reading the manuscript.

\section{ADDED IN PROOF}

Before receiving the galley proofs, we became aware of A. G. Baskakov's work Wiener's theorem and asymptotic estimates for elements of inverse matrices in Funktsional. Anal. i Prilozhen 24 (1990), 64-65. It contains a version of our Corollary 11 with a completely different proof. We thank Ilya Krystall for pointing out this reference to us. 


\section{REFERENCES}

[1] N. Atreas, J. J. Benedetto, and C. Karinakas. Local sampling for regular wavelet and Gabor expansions. Sampling Th. Signal Image Proc., 2(1):1-24, 2003. MR2002854 (2004k:42050)

[2] R. Balan, P. Casazza, C. Heil, and Z. Landau. Density, redundancy, and localization of frames. II. Preprint, 2005.

[3] B. A. Barnes. The spectrum of integral operators on Lebesgue spaces. J. Operator Theory, 18(1):115-132, 1987. MR0912815 (89i:46065)

[4] B. A. Barnes. Symmetric Banach *-algebras: invariance of spectrum. Studia Math., 141(3):251-261, 2000. MR:1784672 (2001h:46098)

[5] H. Bölcskei and A. J. E. M. Janssen. Gabor frames, unimodularity, and window decay. J. Fourier Anal. Appl., 6(3):255-276, 2000. MR.1755143 (2001b:42044)

[6] L. H. Brandenburg. On identifying the maximal ideals in Banach algebras. J. Math. Anal. Appl., 50:489-510, 1975. MR0377523 (51:13695)

[7] P. G. Casazza. The art of frame theory. Taiwanese J. Math., 4(2):129-201, 2000. MR.1757401 (2001f:42046)

[8] O. Christensen. An introduction to frames and Riesz bases. Applied and Numerical Harmonic Analysis. Birkhäuser Boston, Inc., Boston, MA, 2003. MR1946982(2003k:42001)

[9] O. Christensen and T. Strohmer. The finite section method and problems in frame theory. Preprint, 2003.

[10] E. Cordero and K. Gröchenig. Localization of frames II. Appl. Comput. Harmon. Anal., 17:29-47, 2004. MR2067914

[11] I. Daubechies. Ten lectures on wavelets. Society for Industrial and Applied Mathematics (SIAM), Philadelphia, PA, 1992. MR 1162107 (93e:42045)

[12] S. Demko, W. F. Moss, and P. W. Smith. Decay rates for inverses of band matrices. Math. Comp., 43(168):491-499, 1984. MR0758197(85m:15002)

[13] H. G. Feichtinger. Gewichtsfunktionen auf lokalkompakten Gruppen. Österreich. Akad. Wiss. Math.-Natur. Kl. Sitzungsber. II, 188(8-10):451-471, 1979. MR0599884 (81m:43004)

[14] H. G. Feichtinger and N. Kaiblinger. Perturbation of Gabor frames. Trans. Amer. Math. Soc., 356(5):2001-2023, 2004. MR2031050 (2004k:42044)

[15] G. Fendler, K. Gröchenig, M. Leinert, J. Ludwig, and C. Molitor-Braun. Weighted group algebras on groups of polynomial growth. Math. Z., 102(3):791-821, 2003. MR2020712 (2004k:43008)

[16] I. Gel'fand, D. Raikov, and G. Shilov. Commutative normed rings. Chelsea Publishing Co., New York, 1964. MR0205105 (34:4940)

[17] K. Gröchenig. Foundations of time-frequency analysis. Birkhäuser Boston, Inc., Boston, MA, 2001. MR1843717 (2002h:42001)

[18] K. Gröchenig. Localized frames are finite unions of Riesz sequences. Adv. Comput. Math., 18(2-4):149-157, 2003. MR 1968117 (2004a:42044)

[19] K. Gröchenig. Localization of frames, Banach frames, and the invertibility of the frame operator. J.Fourier Anal. Appl., 10(2):105-132, 2004. MR2054304

[20] K. Gröchenig and M. Leinert. Wiener's lemma for twisted convolution and Gabor frames. J. Amer. Math. Soc., 17:1-18, 2004. MR2015328 (2004m:42037)

[21] R. Hagen, S. Roch, and B. Silbermann. $C^{*}$-algebras and numerical analysis, volume 236 of Monographs and Textbooks in Pure and Applied Mathematics. Marcel Dekker, Inc., New York, 2001. MR1792428 (2002g:46133)

[22] C. E. Heil and D. F. Walnut. Continuous and discrete wavelet transforms. SIAM Rev., 31(4):628-666, 1989. MR1025485 (91c:42032)

[23] A. Hulanicki. On the spectrum of convolution operators on groups with polynomial growth. Invent. Math., 17:135-142, 1972. MR0323951(48:2304)

[24] S. Jaffard. Propriétés des matrices "bien localisées" près de leur diagonale et quelques applications. Ann. Inst. H. Poincaré Anal. Non Linéaire, 7(5):461-476, 1990. MR1138533 (93h:47035)

[25] V. Losert. On the structure of groups with polynomial growth. II. J. London Math. Soc. (2), 63(3):640-654, 2001. MR1825980 (2002f:22007)

[26] T. W. Palmer. Spectral algebras. Rocky Mountain J. Math., 22(1):293-328, 1992. MR1159960 (93d:46079) 
[27] T. W. Palmer. Banach algebras and the general theory of ${ }^{*}$-algebras. Vol. I, volume 49 of Encyclopedia of Mathematics and its Applications. Cambridge University Press, Cambridge, 1994. MR.1270014 (95c:46002)

[28] T. W. Palmer. Banach algebras and the general theory of $*$-algebras. Vol. 2, volume 79 of Encyclopedia of Mathematics and its Applications. Cambridge University Press, Cambridge, 2001. MR1819503 (2002e:46002)

[29] T. Pytlik. On the spectral radius of elements in group algebras. Bull. Acad. Polon. Sci. Sér. Sci. Math. Astronom. Phys., 21:899-902, 1973. MR0328476 (48:6818)

[30] M. Reed and B. Simon. Methods of modern mathematical physics. I. Academic Press, Inc. [Harcourt Brace Jovanovich, Publishers], New York, second edition, 1980. MR0751959 (85e:46002)

[31] W. Rudin. Functional analysis. McGraw-Hill Series in Higher Mathematics. McGraw-Hill Book Co., New York, 1973. MR0365062 (51:1315)

[32] J. Sjöstrand. Wiener type algebras of pseudodifferential operators. Séminaire sur les Équations aux Dérivées Partielles, 1994-1995, Exp. No. IV, 21, École Polytech., Palaiseau, 1995. MR.1362552 (96j:47049)

[33] T. Strohmer. Rates of convergence for the approximation of shift-invariant systems in $\ell^{2}(\mathbb{Z})$. J. Fourier Anal. Appl., 5(6):599-616, 2000. MR1752593 (2001b:42041)

[34] T. Strohmer. Approximation of dual Gabor frames, window decay, and wireless communications. Appl. Comput. Harmon. Anal., 11(2):243-262, 2001. MR1848305 (2002j:42049)

[35] T. Strohmer. Four short stories about Toeplitz matrix calculations. Linear Algebra Appl., 343/344:321-344, 2002. Special issue on structured and infinite systems of linear equations. MR1878948 (2002k:47060)

Department of Mathematics, The University of Connecticut, Storrs, Connecticut 06269-3009

E-mail address: groch@math.uconn.edu

Current address: Faculty of Mathematics, University of Vienna, Nordbergstrasse 15, A-1090 Vienna, Austria

E-mail address: karlheinz.groechenig@univie.ac.at

Fakultät für Mathematik, Institut für Angewandte Mathematik, Im Neuenheimer Feld 288, D-69120 Heidelberg, Germany

E-mail address: leinert@math.uni-heidelberg.de 